\title{
On vibration mitigation and energy harvesting of a non- ideal system with autoparametric vibration absorber system
}

\author{
Jorge Luis Palacios Felix • Jose Manoel Balthazar • Rodrigo Tumolin Rocha • \\ Angelo Marcelo Tusset · Frederic Conrad Janzen
}

Received: 23 October 2017/Accepted: 12 July 2018/Published online: 18 July 2018

(C) Springer Nature B.V. 2018

\begin{abstract}
This paper demonstrates that vibration mitigation and energy harvesting can be achieved simultaneously by using of an electricity-generating from autoparametric vibration absorber system (AVAS) and non-ideal system (NIS). The NIS consists of a simple portal frame excited by a small dc motor with eccentric mass, with limited power supply and located on the top. The AVAS consists of a cantilever beam with tip mass parallel coupled to NIS. A piezoelectric material is considered for energy harvesting installed in the base of the AVAS and an
\end{abstract}

J. L. P. Felix

UFFS - Federal University of Fronteira Sul, Cerro Largo, RS 97900-000, Brazil

e-mail: jorge.felix@uffs.edu.br

\section{J. M. Balthazar}

Faculty of Mechanical Engineering, UNESP - Sao Paulo State University, CP 473, Bauru, SP 17033-360, Brazil

e-mail: jmbaltha@ita.br

J. M. Balthazar

ITA - Aeronautics Technological Institute,

Sao Jose dos Campos, SP 12228-900, Brazil

R. T. Rocha $(\varangle)$ · A. M. Tusset · F. C. Janzen UTFPR - Federal University of Technology - Parana, Ponta Grossa, PR 84016-210, Brazil

e-mail: digao.rocha@gmail.com
A. M. Tusset
e-mail: tusset@utfpr.edu.br
F. C. Janzen
e-mail: fcjanzen@utfpr.edu.br

electric circuit is connected to the piezoelectric material in order to produce voltage output. Several numerical simulations were carried out focusing on the passage through the resonance of NIS, when the motor rotational frequency is near the portal frame natural frequency and when the non-ideal subsystem frequency is approximately twice the absorber beam frequency (two-to-one internal resonance). The results showed the existence of Sommerfeld effect in NIS and saturation phenomenon in the NIS-AVAS.

Keywords Energy harvesting - Autoparametric absorber $\cdot$ Non-ideal vibration $\cdot$ Saturation phenomenon

\section{Introduction}

In the last few decades, the interest in study autoparametric vibration absorbers for mechanical structural systems has been widely increased. Small-scale laboratory models were tested to gain understanding of the numerous requirements for optimum performance of these vibration suppression devices. They have many practical implementations in civil and mechanical engineering. Vibrations of coupled systems are modeled in terms of nonlinear ordinary differential equations. Such systems contain different types of nonlinearities. One common type of nonlinearity which is observed is the quadratic nonlinearity. 
Systems with such nonlinearities possess some characteristic features. Two-to-one internal resonances occur frequently and energy is easily transferred from the excited mode to the specific mode with 2:1 internal resonance. Different physical systems of autoparametric absorber were presented in [1]. The authors of [2] devised an autoparametric vibration absorber (AVAS) by attaching a cantilever beam with a tip mass to a linear spring mass system under external forcing. Their experimental validation showed that such an absorber is very effective in suppressing the vibrations of the base structure.

Moreover, recently, there is a great interest in the process of Energy Harvesting. In this process, electrical energy is obtained through the conversion of mechanical energy from an ambient vibration. The vibrations, i.e., kinetic energy is in many different applications and readily available in the daily of human kind. In addition, this energy source is widely wasted because some problems which vibration may cause. Therefore, it is purposed their extraction. A newest and promising way to harvest the vibration energy is using piezoelectric materials described by many authors, and showing the actuality of the energy harvesting theme [3-9]. The extraction of energy from a vibrating environment which is under direct force or/ and base excitation with maximum power flow occurs when the frequency of excitation is equal to the undamped natural frequency and employing a permanent magnetic DC motor/generator.

On the other hand, the study of non-ideal vibrations has drawn special attention of engineering researchers in recent years. Consider an unbalanced motor on an elastic foundation. If it is considered the region before resonance in the Frequency-Response curve of this system, it is noted that as the power supplied to the motor increases, its speed of rotation increases accordingly. However, this behavior does not continue indefinitely. The closer the motor speed moves toward the resonant frequency, the more power is required to increase the motor speed, as part of the energy is consumed moving the supporting structure. A large change in the power supplied to the motor results in a small change in its frequency and a large increase in the amplitude of the resulting elastic support oscillations. Therefore, near resonance, it appears that the additional power supplied to the motor only increases the amplitude of the response of the supporting structure while there is a little effect on the RPM of the motor. Eventually, enough power is supplied to the motor to initiate the jump, the operating frequency increases and the amplitude decreases, resulting in lower power consumption by the motor [10-19].

The resulting responses can include energy transfer from one mode of the system to another one, which is a phenomenon called saturation. Such phenomenon was studied in non-ideal problems depending on the physical and geometric properties of the portal frame to tune the natural frequencies of the two main modes into a 1:2 internal resonance and the non-ideal excitation frequency set near of the second natural frequency [20-22]. The implementation of the active saturation control to suppress steady-state vibrations of a non-ideal simple portal frame structure was proposed by [23] connecting it to a second-order controller using quadratic position coupling terms. Saturation active control method was initially proposed and studied in [24-27]. Other example of these systems that saturation phenomenon was applied are the L-shaped beam structure by [28, 29] and the nonlinear saturation controller was applied for nonlinear beam by $[30,31]$. The nonlinear energy harvesting material in non-ideal problems with saturation phenomenon was introduced as in [3, 32-36].

Autoparametric vibration absorber has been used to reduce vibrations in single-degree-of-freedom structures [37] and in non-ideal systems [38]. Attaching an energy harvester device to an autoparametric vibration response would be of interest for the purposes of enhancing its control effects and use of the harvested power. Numerical analysis of a pendulum vibration absorber was used with a device to energy recovery which uses a movable magnet located between two fixed magnets [38]. The energy harvester depends on the autoparametric pendulum-oscillator motion and mounted in the pendulum pivot-rotor [39]. Some researchers have studied a L-shaped beam-mass structure as a new piezoelectric energy harvester configuration tuning parameters. These studies have tried to make possible the obtaining of the two-to-one internal resonance and saturation phenomena for the nonlinear modal interactions and with base excitation [6, 40, 41]. Also, other researchers have studied an inverted T-shape configuration. The primary parametric resonator is the vertically upright cantilever beam resting on a horizontal clamped-clamped initial spring. When a vertical driving force is applied, the initial spring is directly excited while the cantilever can be 
parametrically excited under the auto-parametric resonance conditions (the natural frequency of the initial spring is twice the natural frequency of the cantilever) [42-44].

In this paper, they are described results from ambient vibration energy harvesting by using the parallel coupling between a non-ideal portal frame (NIS) and an autoparametric cantilever beam absorber with piezoelectric fixed on the base of the beam. The NIS and AVAS are tuned to a natural frequency ratio of 2:1 of internal resonance. Such autoparametric internal resonance allows the system to possess vibration energy transfer between the AVAS and NIS, where the non-ideal system is resonantly excited by the DC motor. Thus, the aim of this work is to tune the passive autoparametric vibration absorber to suppress the non-ideal portal frame vibration and generate the maximum vibration of the absorber vertical beam to act as an energy harvester by mean of saturation phenomenon. When the amplitude of the portal frame beam reaches a critical point, its motion saturates and transfer the surplus energy to the AVAS because of the 2:1 internal resonance, which is the saturation phenomenon. With that, the surplus energy transferred to the absorber is converted into electrical energy by means of the piezoelectric material coupled to the beam of the absorber, thus the higher the vibration transferred to the AVAS the higher amount of power will be converted.

Therefore, the next section presents the engineering problem and the mathematical modeling of the nonideal coupled system.

\section{Engineering problem and mathematical model design}

In Fig. 1, it is described a schematic diagram of the energy harvesting/autoparametric non-ideal system. Here, the non-ideal system consists of a simple portal frame of supporting an unbalanced motor with limited power at its top of $\left(x_{1}, m_{1}, \varphi, m_{0}, r, k_{1}, c_{1}\right)$ displacement, mass, angular displacement, unbalanced mass, eccentricity, stiffness and damping. In order attenuate the vibration of the simple portal frame is used a horizontal cantilever beam coupled in the top of the simple portal frame with tip mass and piezoelectric material fixed in the base of the beam of $\left(x_{2}, m_{2}, k_{2}, c_{2}\right)$ displacement, mass, stiffness and damping.

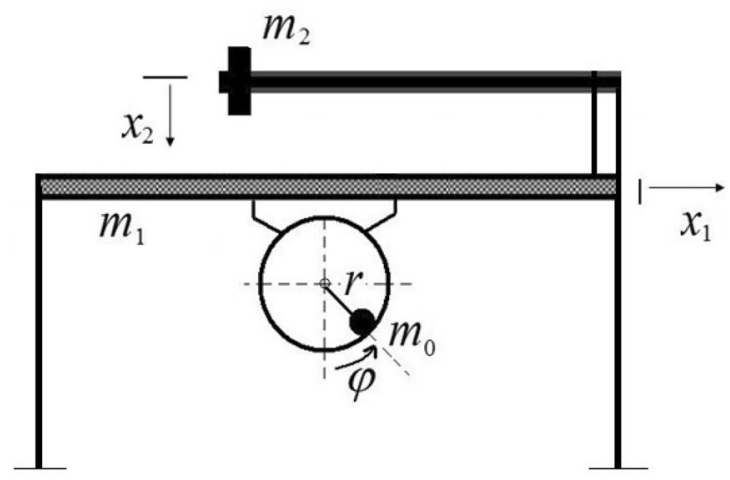

(a)

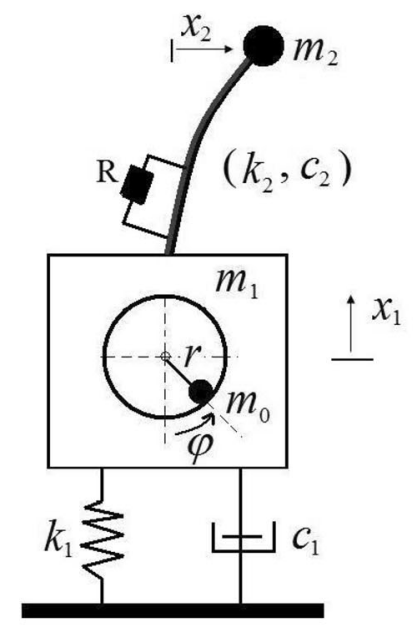

(b)

Fig. 1 Schematic diagram of autoparametric non-ideal system and energy harvester, a physical model, $\mathbf{b}$ mathematical model

The motion in the horizontal direction $x_{1}$ of the simple portal frame is excited with the motor torque. The torque of the motor, denoted by Eq. (1) contains two terms: the characteristic of the motor $L(\dot{\varphi})$ and the resisting moment $H(\dot{\varphi})$ due primarily to windage of the rotating parts outside the motor

$\Gamma(\dot{\varphi})=L(\dot{\varphi})-H(\dot{\varphi})$.

Generally, $L(\dot{\varphi})$ and $H(\dot{\varphi})$ are determined experimentally. It is assumed the motor torque as a linear function of the angular velocity (speed of rotation) $\dot{\varphi}$ in the form of Eq. (2)

$\Gamma(\dot{\varphi})=\alpha-\beta \dot{\varphi}$,

where $\alpha$ is the constant corresponding the voltage applied to the motor and $\beta$ is a constant for each model 
of the DC motor considered (or corresponding to the difference between the torque generated by the motor and the resistance torque).

The governing equations of motion of the non-ideal portal frame are given by Eqs. (3) and (5) considered as the simplified model of [23], the modeling of the autoparametric vibration absorber is given by Eq. (4) proposed by [36] and the modeling of the piezoelectric energy harvester is given by Eq. (6) considered as in [6]

$$
\begin{aligned}
& \left(m_{0}+m_{1}+m_{2}\right) \ddot{x}_{1}+c_{1} \dot{x}_{1}+k_{1} x_{1}-\eta_{1}\left(x_{2} \ddot{x}_{2}+\dot{x}_{2}^{2}\right) \\
& \quad=m_{0} r\left(\ddot{\varphi} \sin \varphi+\dot{\varphi}^{2} \cos \varphi\right),
\end{aligned}
$$

$\ddot{x}_{2}+\frac{c_{2}}{m_{2}} \dot{x}_{2}+\frac{k_{2}}{m_{2}} x_{2}+\eta_{2}\left(x_{2} \ddot{x}_{2}+\dot{x}_{2}^{2}\right) x_{2}-\theta v=\eta_{1} x_{2} \ddot{x}_{1}$,

$$
\left(I+m_{0} r^{2}\right) \ddot{\varphi}=\Gamma(\dot{\varphi})+m_{0} r \ddot{x}_{1} \sin \varphi,
$$

$C_{p} \dot{v}+\frac{1}{R} v+\Theta \dot{x}_{2}=0$,

where $R$ is the load resistance; $C_{p}$ is the capacitance of the piezoelectric layer; $\theta$ and $\Theta$ are the electromechanical coupling coefficients that depending of material constant and design of energy harvester. Where $\eta_{1}$ and $\eta_{2}$ are coupling constants.

To guarantee the autoparametric interaction between the first mode of the non-ideal portal frame and the cantilever beam absorber, by which the attenuation is obtained, the following two-mode autoparametric tuning conditions must be satisfied

$\Omega=\omega_{1}$,

$\omega_{1}=2 \omega_{2}$,

where $\Omega$ is the non-ideal excitation frequency, $\omega_{1}$ corresponds the natural frequency of the simple portal frame and $\omega_{2}$ is the natural frequency of the cantilever beam vibration absorber, being that

$$
\begin{aligned}
& \omega_{1}=\sqrt{\frac{k_{1}}{m_{0}+m_{1}+m_{2}}}, \\
& \omega_{2}=\sqrt{\frac{k_{2}}{m_{2}}} .
\end{aligned}
$$

These two expressions of Eqs. (7) and (8) are wellknown as the external and internal resonance conditions, respectively [15].

For convenience, it is taken the static displacement of the simple portal frame as

$$
x_{0}=\frac{\left(m_{0}+m_{1}+m_{2}\right) g}{k_{1}} \text {. }
$$

The dimensionless displacements of the NIS, AVAS and dimensionless time as

$$
\begin{aligned}
& u_{1}=\frac{x_{1}}{x_{0}}, \quad u_{2}=\frac{x_{2}}{x_{0} \sqrt{m_{0}+m_{1}+m_{2}}}, \quad \tau=\omega_{1} t \\
& u_{3}=\frac{v}{v_{0}}
\end{aligned}
$$

and the dimensionless parameters are

$$
\begin{aligned}
\delta & =x_{0} \eta_{1}, \quad \rho=\frac{\eta_{2}\left(m_{0}+m_{1}+m_{2}\right)}{\eta_{1}^{2}}, \quad \omega=\frac{\omega_{2}}{\omega_{1}}, \\
\mu_{1} & =\frac{c_{1}}{\omega_{1}\left(m_{0}+m_{1}+m_{2}\right)}, \quad \gamma_{1}=\frac{m_{0} r}{x_{0}\left(m_{0}+m_{1}+m_{2}\right)}, \\
\mu_{2} & =\frac{c_{2}}{m_{2} \omega_{1}}, \quad \gamma_{2}=\frac{m_{0} r x_{0}}{I+m_{0} r^{2}}, \quad \lambda=\frac{1}{R C_{p} \omega_{1}}, \\
\chi_{1} & =\frac{\theta v_{0}}{x_{0} \omega_{1}^{2} \sqrt{m_{0}+m_{1}+m_{2}}}, \quad \Gamma(\dot{\varphi})=a-b \dot{\varphi}, \\
\chi_{2} & =\frac{\Theta x_{0} \sqrt{m_{0}+m_{1}+m_{2}}}{v_{0} C_{p}}, \quad a=\frac{\alpha}{\left(I+m_{0} r^{2}\right) \omega_{1}^{2}}, \\
b & =\frac{\beta}{\left(I+m_{0} r^{2}\right) \omega_{1}} .
\end{aligned}
$$

Equations (3)-(6) are rewritten in dimensionless form

$$
\begin{aligned}
& \ddot{u}_{1}+\mu_{1} \dot{u}_{1}+u_{1}-\delta\left(u_{2} \ddot{u}_{2}+\dot{u}_{2}^{2}\right) \\
& \quad=\gamma_{1}\left(\ddot{\varphi} \sin \varphi+\dot{\varphi}^{2} \cos \varphi\right), \\
& \ddot{u}_{2}+\mu_{2} \dot{u}_{2}+\omega^{2} u_{2}+\rho \delta^{2}\left(u_{2} \ddot{u}_{2}+\dot{u}_{2}^{2}\right) u_{2}, \\
& \quad-\chi_{1} u_{3}=\delta u_{2} \ddot{u}_{1}, \\
& \ddot{\varphi}=\Gamma(\dot{\varphi})+\gamma_{2} \ddot{u}_{1} \sin \varphi, \\
& \dot{u}_{3}+\lambda u_{3}+\chi_{2} \dot{u}_{2}=0 .
\end{aligned}
$$

The calculation of the harvested power of the autoparametric absorber is given by Eqs. (17) and (18) that describe the dimension and dimensionless harvested power, respectively 


$$
\begin{aligned}
& P=\frac{v^{2}}{R}, \\
& P=R_{0} u_{3}^{2},
\end{aligned}
$$

where $R_{0}=v_{0}^{2} / R$.

\section{Data and state-space notation}

In this section, the numerical results are carried out and presented to the proposal of this work.

The numerical simulations were carried out adopting the 4th order Runge-Kutta method with fixed step, and are given using the parameters of Table 1, which are similar approximation values of [6, 23]. Furthermore, those parameters are considered as standard in the whole work, except when indicated in the text.

The state-space form of the variables are given by Eq. (19)

$$
X=\left[\begin{array}{lllllll}
u_{1} & \dot{u}_{1} & u_{2} & \dot{u}_{2} & \varphi & \dot{\varphi} & u_{3}
\end{array}\right] .
$$

Then, transforming Eqs. (13)-(16) into state-space form, they will have the following form

Table 1 Dimensionless parameters for the system of Eqs. (13)-(16)

\begin{tabular}{lll}
\hline Parameters & Values & Means \\
\hline$\mu_{1}$ & 0.1 & Beam damping \\
$\mu_{2}$ & 0.007 & Absorber damping \\
$\delta$ & 3.1 & Mechanical coupling of the beam \\
$\rho$ & 6.2 & Mechanical coupling of the absorber \\
$\gamma_{1}$ & 0.001 & Electric motor coupling \\
$\gamma_{2}$ & 13.33 & Electric of beam coupling \\
$a$ & 0.545 & Constant torque of the motor \\
$b$ & 0.5 & Angular speed constant of the motor \\
$\chi_{1}$ & 0.03 & Coupling constant of the absorber \\
$\chi_{2}$ & 0.05 & Coupling constant of the piezoelectric \\
$\lambda$ & 0.01 & Stiffness of the piezoelectric \\
$\omega$ & 0.5 & Natural frequency of the absorber \\
$R_{0}$ & 1.0 & Dimensionless power constant \\
\hline
\end{tabular}

$$
\begin{aligned}
& \dot{X}_{1}=X_{2}, \\
& \dot{X}_{2}=F_{1}, \\
& \dot{X}_{3}=X_{4}, \\
& \dot{X}_{4}=F_{2}, \\
& \dot{X}_{5}=X_{6}, \\
& \dot{X}_{6}=F_{3}, \\
& \dot{X}_{7}=-\lambda X_{7}-\chi_{2} X_{4},
\end{aligned}
$$

where

$$
\left(\begin{array}{c}
F_{1} \\
F_{2} \\
F_{3}
\end{array}\right)=A^{-1}\left(\begin{array}{c}
f_{1} \\
f_{2} \\
\Gamma\left(X_{6}\right)
\end{array}\right),
$$

where the matrix $A, f_{1}$ and $f_{2}$ are

$$
\begin{aligned}
& A=\left(\begin{array}{ccc}
1 & -\delta X_{3} & -\gamma_{1} \sin X_{6} \\
-\delta X_{3} & 1+\rho \delta^{2} X_{3}^{2} & 0 \\
-\gamma_{2} \sin X_{6} & 0 & 1
\end{array}\right), \\
& f_{1}=-\mu_{1} X_{2}-X_{1}+\delta X_{4}^{2}+\gamma_{1} X_{6}^{2} \cos X_{6}, \\
& f_{2}=-\mu_{2} X_{4}-\omega^{2} X_{3}-\rho \delta^{2} X_{3} X_{4}^{2}+\chi_{1} X_{7} .
\end{aligned}
$$

Therefore, the next subsection will show the numerical results provided by the governing equations of motion of the system Eq. (20).

\section{Autoparametric non-ideal system numerical results}

The simulations presented in this section are without the coupling of the energy harvesting material. Therefore, in the following it will be presented some discussions about numerical simulations of the considered autoparametric non-ideal system.

Figure 2 presents an example for case $a=0.545$ of resonance capture (Sommerfeld effect) where the angular velocity (speed of rotation) is in resonance with natural frequency of the non-ideal beam $\left(X_{6}=\dot{\varphi} \approx 1\right)$.

Figure 3 presents the numerically simulated time domain response of both non-ideal simple portal frame $\left(X_{1}\right)$ and the autoparametric vibration absorber $\left(X_{3}\right.$ controller).

The control technique based on the saturation phenomenon suppressed the high amplitude of the 


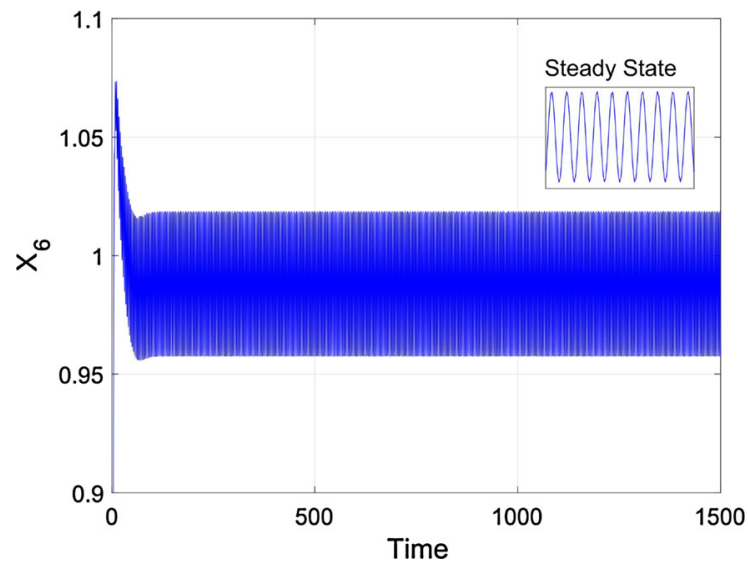

Fig. 2 Angular velocity for the case with resonance capture

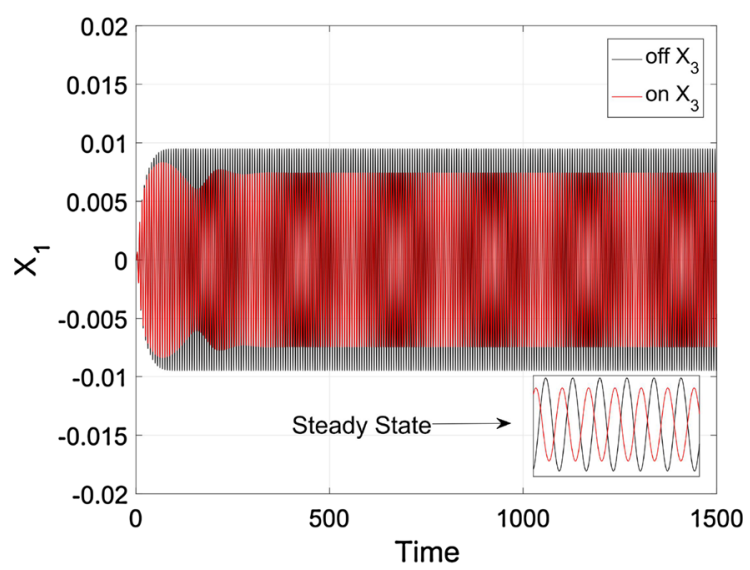

(a)

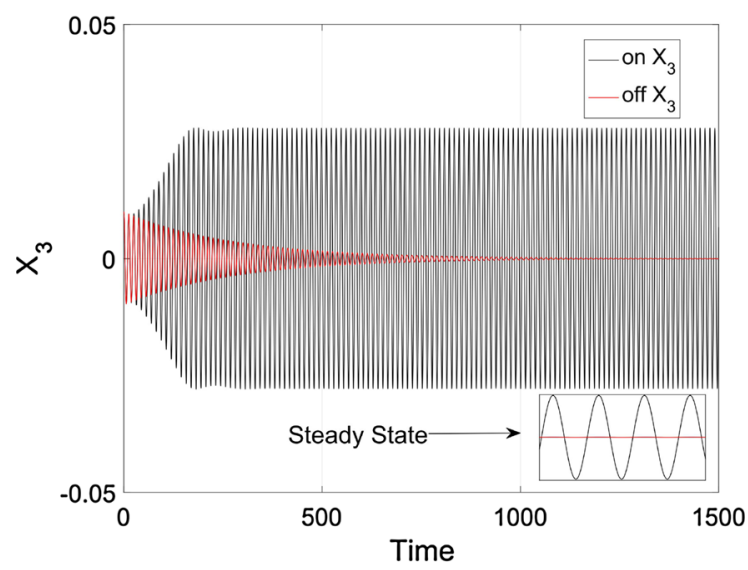

(b)

Fig. 3 Displacement with saturation phenomenon of the a NIS and $\mathbf{b}$ AVAS

first-mode vibration of a reduced non-ideal vertical beam. Because of the two-to-one internal resonance condition kept between the non-ideal portal frame and the cantilever beam absorber $\left(\omega=0.5 \leftrightarrow \omega_{1}=2 \omega_{2}\right)$, the amplitude of the non-ideal beam becomes saturated and its vibrational energy in excess was partiallytransferred to the horizontal beam in the time range $50 \leq \tau \leq 1500$.

Since the control technique suppressed the high amplitudes of the non-ideal portal frame and the autoparametric cantilever beam absorber becomes higher than the NIS, a piezoelectric material was coupled to harvest energy from the vibration energy of the AVAS, which will be detailed in the next subsection.

\section{Energy harvesting strategy numerical results}

Figure 4a shows the steady-state responses of the system, represented by Eqs. (13)-(16), during the passage through fundamental resonance region ( $\dot{\varphi} \approx 1$ ) which corresponds to the output of $X_{6}$ in a variation of a designated control parameter $a$ with an amplitude diagram. However, in Fig. 4b, they are shown the amplitudes of each coordinate $X_{1}, X_{3}$ and $X_{7}$. Each amplitude consists of the maximum absolute value of the amplitude oscillation of $X_{1}, X_{3}$ and $X_{7}$ that are the output of the non-ideal portal frame, autoparametric cantilever beam absorber, and the harvested voltage, respectively.

The control parameter $a$, depending on the voltage applied to motor, it is applied in the range $0.48 \leq a \leq 0.6, \quad$ considering an increment $\Delta a=0.001$. The numerical simulations were carried out in the dimensionless time domain $0 \leq \tau \leq 1500$.

Figure 4a shows the Sommerfeld effect. It is noted that as the power supplied to the motor increases, its speed of rotation (angular velocity $\dot{\varphi}$ ) increases indefinitely on $0.48 \leq a \leq 0.6$.

The closer the motor speed moves toward the resonant frequency $(\dot{\varphi} \approx 1)$, the more power is required to increase the motor speed, as part of the energy is consumed moving the simple portal frame. A large change in the power supplied to the motor results in a small change in its frequency and a large increase in the amplitude of the resulting elastic support oscillations. Thus, passage through resonance is observed in $0.54 \leq a \leq 0.566$. 


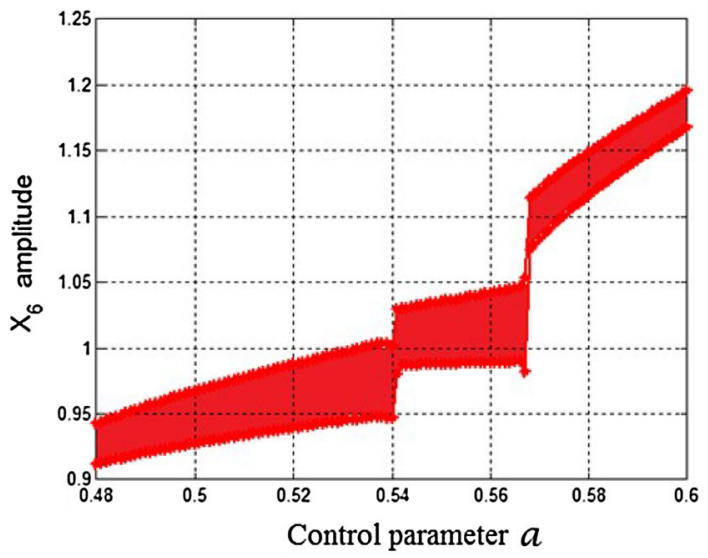

(a)

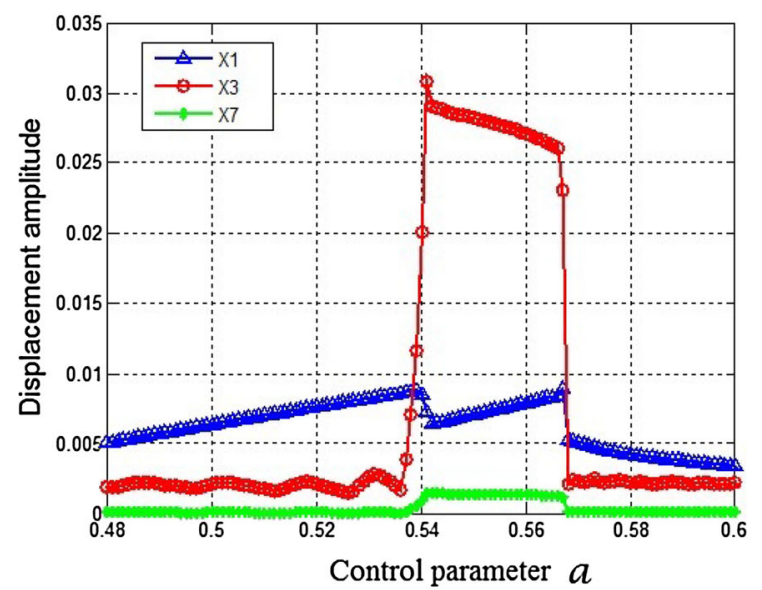

(b)

Fig. 4 a Sommerfeld effect of $X_{6}$ (angular velocity); b saturation phenomenon in $X_{1}$ (NIS), $X_{3}$ (AVAS) and $X_{7}$ voltage

With the additional power supplied to the motor, it is observed that the angular velocity continues to be captured in the resonance range while the amplitude of the response of the simple portal frame is saturated and then transferred to the autoparametric cantilever beam absorber (saturation phenomenon), as it is possible to see in Fig. 4b.

Due to the internal resonance ratio and the external resonance range between the motor and NIS saturation phenomenon occurs, and the energy harvesting is possible as shown the harvested power versus $a$, in Fig. 5. In the resonance range, the amount of harvested power is improved from approximately zero to an interval of $1.2<P<2.5\left[10^{-6}\right]$.

Saturation phenomenon depends directly on the internal and external resonances configurations. With

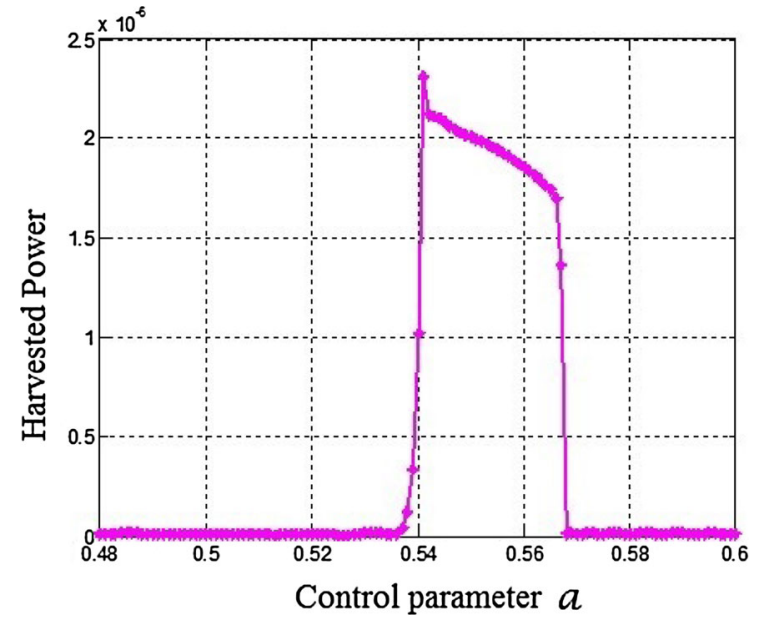

Fig. 5 Electric power from AVAS with variation non-ideal torque

saturation, the energy harvesting is possible with a great increase in the harvested power. Thus, the next section investigates mutual influences of the motor parameters $a$ and $b$, with the natural frequency of $u_{2}$.

\section{Influence of the motor-frequency parameters in the autoparametric non-ideal system}

It is very important to highlight what are the contributions of the motor to the suppression of the portal frame amplitude. The joint analysis of the motor torque $a$, the angular speed constant $b$ and the natural frequency of the absorber $\omega$ depicts an overview of the three parameters related to the involved phenomena and energy harvesting. Thus, the amplitudes of the portal frame and autoparametric vibration absorber beam with piezoceramic layers along this such beam [6] and the harvested power versus those parameters will be carried out and divided into three cases. For all the cases, the default parameters of Table 1 are still considered, except when one or more of them are analyzed.

The first case is for the condition of the internal resonance fixed in $\omega_{1}=2 \omega_{2}$, considering the variation of $0.4 \leq a \leq 0.6$ and $0.2 \leq b \leq 1.0$. Figure $6 a$ and $\mathrm{c}$ show the surfaces of the maximum displacement of the portal frame $u_{1}$ and the autoparametric cantilever beam absorber $u_{2}$ in the domain of the motor torque $a$ versus the angular speed of the motor $b$. Both surfaces show that there is a continuous peak in a 


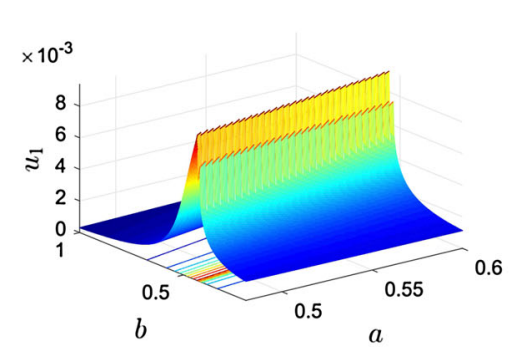

(a)

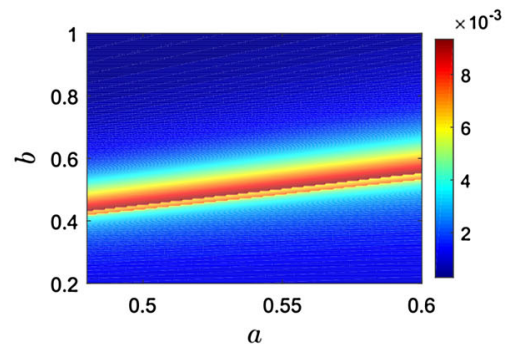

(b)

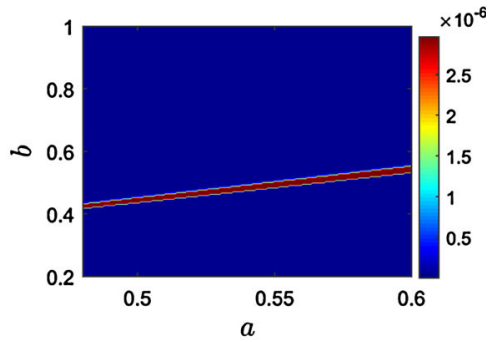

(e)

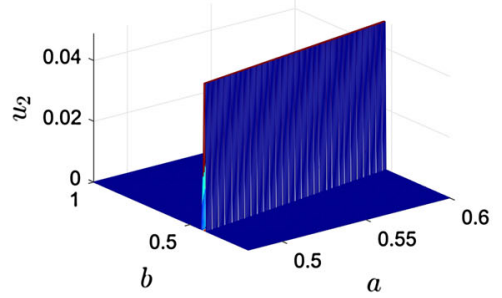

(c)

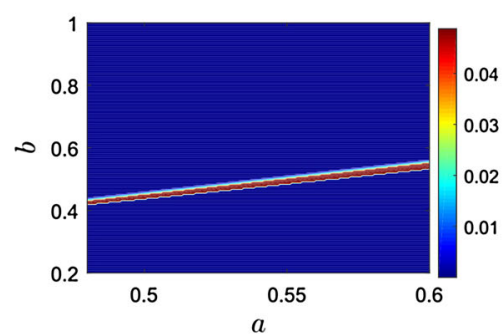

(d)

Fig. 6 Surface of $\mathbf{a} u_{1}$ and $\mathbf{c} u_{2}$; contour of the maximum displacement of $\mathbf{b} u_{1}$ and $\mathbf{d} u_{2}$; $\mathbf{e}$ harvested power $P$ in the parameter domain $(a, b)$

narrow region of assembly of the parameters. Looking over both surfaces, Figs. $6 \mathrm{~b}$ and $\mathrm{d}$ are presented showing the contour of $b$ versus $a$ related to the maximum displacement of $u_{1}$ and $u_{2}$, respectively. The linear area (red colour) reveals the capacity of energy harvesting due to the maximum amplitudes of $u_{2}$ around of 0.04 , which depends on the linear relation between the parameters values $0.4 \leq a \leq 0.6$ and $0.4 \leq b \leq 0.5$. Figure $6 \mathrm{e}$ show that the same coloured region is when the energy harvesting is increased from almost 0 to approximately $3.0 \cdot 10^{-6}$.

The increase in the amplitude of displacement of the absorber is due to the saturation phenomenon occurrence. With that, it was possible to suppress the high undesired amplitude of motion in the portal frame, partially transferring energy to the absorber beam, and, in addition, to harvest energy, as commented in Sec. 5. It is important to highlight that besides the increasing in the amplitude of $u_{2}$, the amplitude of the portal frame also increased due to the external resonance of the system, although saturated.

The second case is for the value of the angular speed of the motor fixed in $b=0.5$, considering the variation of $0.4 \leq a \leq 0.6$ and $0.4 \leq \omega \leq 0.7$. Figure $7 \mathrm{a}$ and $\mathrm{c}$ show the surfaces of the maximum displacement of $u_{1}$ and $u_{2}$, respectively in the domain of the motor torque $a$ and the internal resonance ratio $\omega=\omega_{2} / \omega_{1}$. Looking over the surfaces, Fig. 7b and $d$ show the contour of the $a$ versus $\omega$ related to the maximum displacement of $u_{1}$ and $u_{2}$, respectively. The triangle area (red colour) reveal the capacity of energy harvesting due maximum amplitudes of $u_{2}$ near 0.04 and 0.05 depending on the parameters values in the range $0.54 \leq a \leq 0.58$ and $0.45 \leq \omega \leq 0.51$, approximately. Differently of the first case, the saturation region showed that the amplitude of the portal frame decayed to almost zero whereas the absorber increased up to 0.05 . In addition, it is possible to note that there is a range where the conditions of saturation phenomenon are not satisfied, showing a small discontinuation to the phenomenon, that is when $a \approx 0.56$ and $0.515 \leq \omega \leq 0.535$. After that, saturation occurred again.

With that, Fig. 7e shows that the harvested power increases from almost 0 to approximately $3.5 \cdot 10^{-6}$, higher than the first case.

The third case is for the torque of the motor fixed in $a=0.545$ of resonance capture, considering the variation of $0.2 \leq b \leq 1.0$ and $0.4 \leq \omega \leq 0.7$, Fig. $8 \mathrm{a}$ and $\mathrm{c}$ show surfaces of the maximum displacement of the $u_{1}$ and $u_{2}$, respectively, in the domain of the angular speed of the motor $b$ and the internal resonance ratio $\omega$. Figure $8 \mathrm{~b}$ and $\mathrm{d}$ show the contour of the $b$ versus $\omega$ related to the maximum displacement 


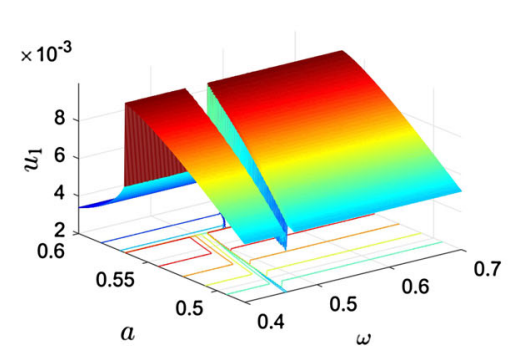

(a)

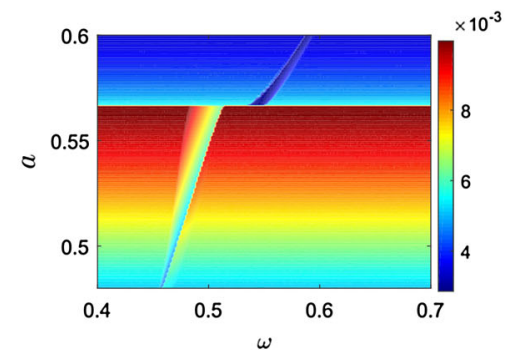

(b)

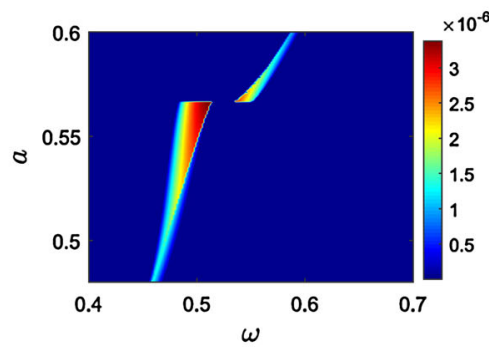

(e)

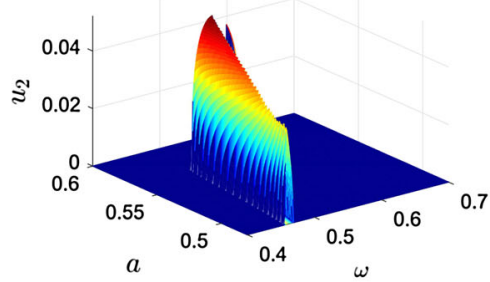

(c)

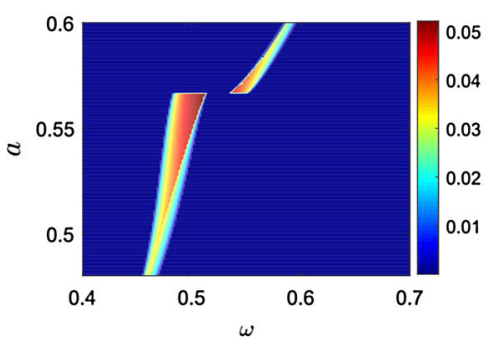

(d)

Fig. 7 Surface of $\mathbf{a} u_{1}$ and $\mathbf{c} u_{2}$; contour of the maximum displacement of $\mathbf{b} u_{1}$ and $\mathbf{d} u_{2}$; $\mathbf{e}$ harvested power $P$ in the parameter domain $(a, \omega)$

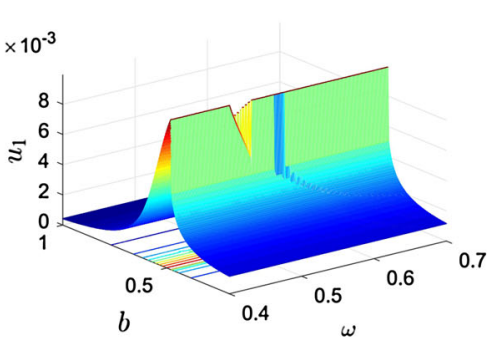

(a)

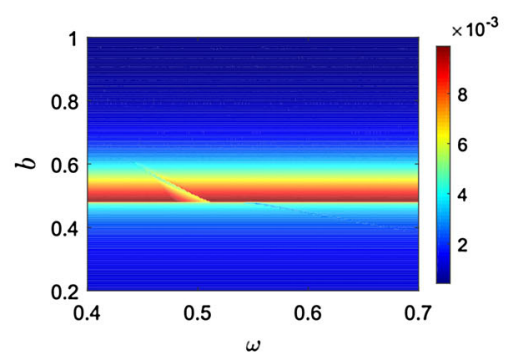

(b)

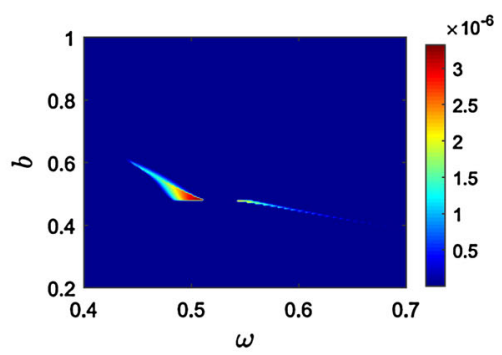

(e)

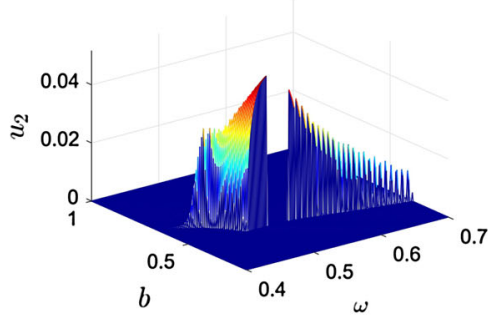

(c)

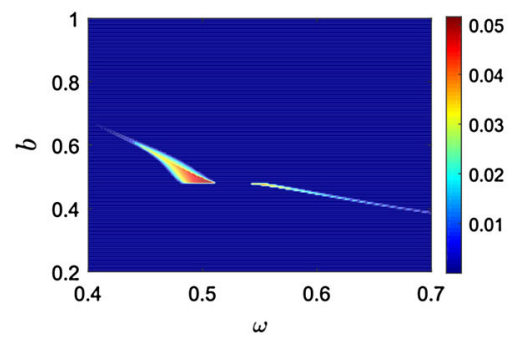

(d)

Fig. 8 Surface of $\mathbf{a} u_{1}$ and $\mathbf{c} u_{2}$; contour of the maximum displacement of $\mathbf{b} u_{1}$ and $\mathbf{d} u_{2}$; $\mathbf{e}$ harvested power $P$ in the parameter domain $(b, \omega)$

of $u_{1}$ and $u_{2}$, respectively. The triangle area (red colour) reveal the capacity of energy harvesting due maximum amplitudes of $u_{2}$ in the range 0.04 and 0.05 depending on the parameters values of $b$ around of 0.5 and $\omega$ around of 0.5 , very close. Similarly to the second case, saturation phenomenon showed to be stronger (higher amplitude) with $\omega \approx 0.5$, however, it presented the same discontinuity when $b \approx 0.48$ and $0.51 \leq \omega \leq 0.542$. After that, saturation continued, although weak (lower amplitude). 
Figure 8e shows the harvested power increases from almost 0 to approximately $3.2 \cdot 10^{-6}$, near to the second case.

With these analyses, some conclusions can be taken out.

\section{Conclusions}

In summary, this paper shows the investigation of the dynamical behaviour of the coupling between nonideal simple portal frame and autoparametric cantilever beam absorber with piezoelectric material fixed in the base of the AVAS. The NIS and AVAS are tuned to a natural frequency ratio of $2: 1$. The aim is to tune the absorber to cancel the non-ideal simple portal frame vibration and produce maximum vibration of the cantilever beam to act as an energy harvester.

With the natural frequency ratio 2:1, the system was set to a two-to-one internal resonance which is an important condition to saturation phenomenon occurs. When the unbalanced motor was in resonance with the non-ideal portal frame, the saturation occurred and transferred part of its vibrating energy to the horizontal beam (AVAS), making possible to harvest energy from its vibration.

The linear torque of the motor was set as a control parameter to a general analysis of the resonance region. Moreover, Sommerfeld effect was detected. With the maintain of resonance from the Sommerfeld effect, the electric power output had wide bandwidth.

In the investigation of the motor-frequency parameters, it was possible to see that saturation appears in a small area, however, being worthy to study, for there is a high increase of the amplitude of displacement of $u_{2}$, decreasing the amplitude of $u_{1}$. It made possible to harvesting energy considering the saturation phenomenon configuration. The second case showed to be the most effective in change the topology of the system, i.e., the torque of the motor has a foremost influence in the external frequency, thus, reaching easily the resonance of the system. However, it will depend on the angular speed of the motor, which is directly applied to the frequency of the motor. Considering both interaction of $a$ and $b$, as in first case, they possess a linear influence in the external frequency, i.e., they reaches the external frequency in
0.5 at any time, depending on both values at the same time.

The theoretical study of this paper supports a future works that may consider experimental assembly.

Acknowledgements The authors acknowledge support by FAPERGS, CNPq, CAPES and FAPESP, all Brazilian research funding agencies.

\section{References}

1. Nayfeh AH (2000) Nonlinear interactions: analytical, computational, and experimental methods. Wiley, New York

2. Haxton RS, Barr ADS (1972) The autoparametric vibration absorber. J Eng Ind 94(1):119-125. https://doi.org/10.1115/ 1.3428100

3. Rocha RT (2016) On saturation phenomenon in energy harvesting based on nonlinear piezoelectric materials coupled to a portal frame foundation with ideal and non-ideal excitations. Ph.D. Dissertation, 2016

4. Preumont A (2006) Mechatronics: dynamics of electromechanical and piezoelectric systems, vol 136. Springer, Berlin

5. Priya S, Inman DJ (2009) Energy harvesting technologies, vol 21. Springer, New York

6. Erturk A, Hoffmann J, Inman DJ (2009) A piezomagnetoelastic structure for broadband vibration energy harvesting. Appl Phys Lett 94(25):254102. https://doi.org/10.1063/ 1.3159815

7. Erturk A, Inman DJ (2011) Piezoelectric energy harvesting. Wiley, Hoboken

8. Litak G, Friswell MI, Kwuimy CAK, Adhikari S, Borowiec M (2012) Energy harvesting by two magnetopiezoelastic oscillators with mistuning. Theor Appl Mech Lett 2(4):043009. https://doi.org/10.1063/2.1204309

9. Stephen NG (2006) On energy harvesting from ambient vibration. J Sound Vib 293(1):409-425. https://doi.org/10. 1016/j.physd.2010.01.019

10. Kononenko VO (1969) Vibrating systems with limited power supply. Illife Books, London

11. Balthazar JM, Mook DT, Weber HI, Brasil RMLRF, Fenili A, Belato D, Felix JLP (2003) An overview on non-ideal vibrations. Meccanica 38(6):613-621. https://doi.org/10. 1023/A:1025877308510

12. Felix JLP, Balthazar JM, Brasil RMLRF (2009) Comments on nonlinear dynamics of a non-ideal Duffing-Rayleigh oscillator: numerical and analytical approaches. J Sound Vib 319(3):1136-1149. https://doi.org/10.1016/j.jsv.2008. 06.036

13. Warminski J, Balthazar JM, Brasil RMLRF (2001) Vibrations of a non-ideal parametrically and self-excited model. J Sound Vib 245(2):363-374. https://doi.org/10.1006/jsvi. 2000.3515

14. Samantaray AK, Dasgupta SS, Bhattacharyya R (2010) Sommerfeld effect in rotationally symmetric planar dynamical systems. Int J Eng Sci 48(1):21-36. https://doi. org/10.1016/j.ijengsci.2009.06.005 
15. Nayfeh AH, Mook DT (2008) Nonlinear oscillations. Wiley, Hoboken

16. Bisoi A, Samantaray AK, Bhattacharyya R (2017) Control strategies for DC motors driving rotor dynamic systems through resonance. J Sound Vib 411:304-327. https://doi. org/10.1016/j.jsv.2017.09.014

17. Bisoi A, Samantaray AK, Bhattacharyya R (2018) Sommerfeld effect in a two-disk rotor dynamic system at various unbalance conditions. Meccanica 53(4-5):681701. https:// doi.org/10.1007/s11012-017-0757-3

18. Bisoi A, Samantaray AK, Bhattacharyya R (2017) Sommerfeld effect in a gyroscopic overhung rotor-disk system. Nonlinear Dyn 88(3):1565-1585. https://doi.org/10.1007/ s11071-017-3329-0

19. Balthazar JM, Tusset AM, Brasil RMLRF, Felix JLP, Rocha RT, Janzen FC, Nabarrete A, Oliveira C (2018) An overview on the appearance of the Sommerfeld effect and saturation phenomenon in non-ideal vibrating systems (NIS) in macro and MEMS scales. Nonlinear Dyn 93:19. https://doi. org/10.1007/s11071-018-4126-0

20. Palacios JLP, Balthazar JM, Brasil RMLRF (2002) On nonideal and non-linear portal frame dynamics analysis using bogoliubov averaging method. J Braz Soc Mech Sci 24(4):257-265. https://doi.org/10.1590/S010073862002000400002

21. Brasil RMLRF, Garzeri FJ, Balthazar JM (2001) An experimental study of the nonlinear dynamics of a portal frame foundation for a non-ideal motor. In: Proceedings of DETC01 ASME 2001 design engineering technical conference and computers and information in engineering conference, pp 9-12

22. Rocha RT, Balthazar JM, Quinn DD, Tusset AM, Felix JLP (2016, August). Non-ideal system with quadratic nonlinearities containing a two-to-one internal resonance. In: ASME 2016 international design engineering technical conferences and computers and information in engineering conference. American Society of Mechanical Engineers, pp V008T10A015-V008T10A015. https://doi.org/10.1115/ DETC2016-59372

23. Felix JLP, Balthazar JM, Brasil RMLRF (2005) On saturation control of a non-ideal vibrating portal frame foundation type shear-building. J Vib Control 11(1):121-136. https://doi.org/10.1177/1077546305047656

24. Oueini SS, Nayfeh AH, Golnaraghi MF (1997) A theoretical and experimental implementation of a control method based on saturation. Nonlinear Dyn 13(2):189-202. https://doi. org/10.1023/A:1008207124935

25. Oueini SS (1999) Techniques for controlling structural vibrations. Ph.D. Thesis

26. Pai PF, Schulz MJ (2000) A refined nonlinear vibration absorber. Int J Mech Sci 42(3):537-560. https://doi.org/10. 1016/S0020-7403(98)00135-0

27. Pai PF, Wen B, Naser AS, Schulz MJ (1998) Structural vibration control using PZT patches and non-linear phenomena. J Sound Vib 215(2):273-296. https://doi.org/10. 1016/S0020-7403(98)00135-0

28. Haddow AG, Barr ADS, Mook DT (1984) Theoretical and experimental study of modal interaction in a two-degree-offreedom structure. J Sound Vib 97(3):451-473. https://doi. org/10.1016/0022-460X(84)90272-4
29. Bux SL, Roberts JW (1986) Non-linear vibratory interactions in systems of coupled beams. J Sound Vib 104(3):497-520. 460X(86)90304-4

30. Hamed YS, Amer YA (2014) Nonlinear saturation controller for vibration supersession of a nonlinear composite beam. J Mech Sci Technol 28(8):2987-3002. https://doi. org/10.1007/s12206-014-0706-1

31. Xu J, Chen Y, Chung KW (2015) An improved time-delay saturation controller for suppression of nonlinear beam vibration. Nonlinear Dyn 82(4):1691-1707. https://doi.org/ 10.1007/s11071-015-2270-3

32. Balthazar JM, Rocha RT, Brasil RMFL, Tusset AM, de Pontes BR, Silveira M (2014) Mode saturation, mode coupling and energy harvesting from ambient vibration in a portal frame structure. In: ASME 2014 international design engineering technical conferences and computers and information in engineering conference. American Society of Mechanical Engineers, pp V008T11A044V008T11A044. https://doi.org/10.1115/DETC2014-34268

33. Rocha RT, Balthazar JM, Tusset AM, Piccirillo V, Felix JLP (2016) Comments on energy harvesting on a 2:1 internal resonance portal frame support structure, using a nonlinear-energy sink as a passive controller. Int Rev Mech Eng (IREME) 10(3):147-156. https://doi.org/10.15866/ ireme.v10i3.8795

34. Iliuk I, Balthazar JM, Tusset AM, Piqueira JRC, de Pontes BR, Felix JLP, Bueno AM (2013) A non-ideal portal frame energy harvester controlled using a pendulum. Eur Phys J Spec Top 222(7):1575-1586. https://doi.org/10.1140/epjst/ e2013-01946-4

35. Iliuk I, Balthazar JM, Tusset AM, Piqueira JR, de Pontes BR, Felix JLP, Bueno AM (2014) Application of passive control to energy harvester efficiency using a nonideal portal frame structural support system. J Intell Mater Syst Struct 25(4):417-429. https://doi.org/10.1177/ 1045389X13500570

36. Lee WK, Cho DS (2000) Damping effect of a randomly excited autoparametric system. J Sound Vib 236(1):23-31. https://doi.org/10.1006/jsvi.2000.2965

37. Sado D, Kot M (2007) Chaotic vibration of an autoparametrical system with a non-ideal source of power. J Theor Appl Mech 45(1):119-131

38. Mitura A, Kecik K (2016) Influences of system parameters on energy harvesting from autoparametric absorber. Numerical research. Vib Phys Syst 27:281-286

39. Kecik K, Borowiec M (2013) An autoparametric energy harvester. Eur Phys J Spec Top 222(7):1597-1605. https:// doi.org/10.1140/epjst/e2013-01948-2

40. Erturk A, Renno JM, Inman DJ (2009) Modeling of piezoelectric energy harvesting from an L-shaped beam-mass structure with an application to UAVs. J Intell Mater Syst Struct 20(5):529-544. https://doi.org/10.1177/ 1045389X08098096

41. Harne RL, Sun A, Wang KW (2016) Leveraging nonlinear saturation-based phenomena in an L-shaped vibration energy harvesting system. J Sound Vib 363:517531. https:// doi.org/10.1016/j.jsv.2015.11.017

42. Cao DX, Leadenham S, Erturk A (2015) Internal resonance for nonlinear vibration energy harvesting. Eur Phys J Spec 
Top 224:28672880. https://doi.org/10.1140/epjst/e201502594-4

43. Jia Y, Seshia AA (2014) An auto-parametrically excited vibration energy harvester. Sens Actuators A 220:69-75. https://doi.org/10.1016/j.sna.2014.09.012
44. Sodano HA, Park G, Inman DJ (2004) Estimation of electric charge output for piezoelectric energy harvesting. Strain 40(2):49-58. https://doi.org/10.1111/j.1475-1305.2004. 00120.x 